\title{
Influencia del tamaño de maceta y la composición de sustrato sobre la calidad de Pongamia pinnata (L.) Pierre
}

\author{
Influence of pot size and substratum composition on quality of Pongamia pinnata
}

(L.) Pierre

\author{
Victoria Rika Kubota $^{1 *}$, Manuel Marino Enciso Gómez ${ }^{1}$ y Mirtha Vera de Ortiz ${ }^{1}$ \\ ${ }^{1}$ Facultad de Ciencias Agrarias, Universidad Nacional de Asunción. San Lorenzo, Paraguay \\ * Autor para correspondencia (victoria.kubota@agr.una.py) \\ Recibido: 15/11/2014; Aceptado: 29/04/2015.
} http://dx.doi.org/10.18004/investig.agrar.2015.junio.65-71

\section{RESUMEN}

Esta investigación tuvo como objetivo principal evaluar la influencia del tamaño de maceta y la composición de sustrato sobre la calidad de plantas de Pongamia pinnata (L.) Pierre y adicionalmente determinar los problemas fitosanitarios y malformaciones que presentan. Se aplicó un esquema factorial $3 \times 3$, siendo los factores composición de sustrato ( $\mathrm{S} 1$, suelo derivado de basalto; S2, suelo derivado de arenisca y S3, suelo hidromórfico) y tamaño de maceta (M1, 5 x $12 \mathrm{~cm}$; M2, 7 x $15 \mathrm{~cm}$ y M3, $20 \times 25 \mathrm{~cm})$. Los tratamientos fueron distribuidos completamente al azar. Los parámetros morfológicos evaluados fueron el índice de esbeltez, la relación entre peso seco aéreo y peso seco radicular y el índice de calidad de Dickson. La determinación de los problemas fitosanitarios y posibles malformaciones fue realizada periódicamente sin considerar los tratamientos, limitándose a su registro y descripción. Los resultados indican que las plantas producidas en macetas de tamaño 7 x $15 \mathrm{~cm}$ fueron las de mayor calidad debido a la mejor relación entre peso seco aéreo y radicular. Si bien aquellas producidas en macetas de tamaño $20 \times 25 \mathrm{~cm}$ arrojaron el índice de calidad de Dickson más elevado, presentaron menor equilibrio entre sistema aéreo y radicular al igual que los tratamientos con maceta de tamaño 5 x $12 \mathrm{~cm}$. No fue verificada la influencia de composición de sustrato. Por otra parte, fueron observados posible albinismo y malformaciones en algunos individuos, además de ataques de larvas de Liriomyza sp.

Palabras clave: Pongamia pinnata, calidad de plantas, parámetros morfológicos, problemas fitosanitarios, malformaciones.

\begin{abstract}
The main aim of this research was to assess plastic plant pot size and substrate composition influence on the quality of Pongamia pinnata (L) Pierre plants and, additionally, to determine phytosanitary problems and malformations present on them. A $3 \times 3$ factorial design was applied, having as factors substratum composition (S1, soil derived from basalt; S2, soil derived from sandstone and S3, hydromorphic soil) and size of plastic plant pot (M1, 5 x $12 \mathrm{~cm}$; M2, 7 x $15 \mathrm{~cm}$ and M3, $20 \times 25$ $\mathrm{cm})$. Treatments were completely randomly distributed. The morphological parameters evaluated were slenderness index, root shoot dry weight ratio and Dickson quality index. Determination of phytosanitary problems and possible malformations were carried out regularly, regardless of treatment type, limited to registration and description. Results show that the highest quality plants were those on treatments with $7 \times 15 \mathrm{~cm}$ plastic plant pots, due to a better shoot dry weight/root dry weight ratio. Although plants on treatment with 20x25 $\mathrm{cm}$ plastic plant pot yielded the highest Dickson quality index, they presented less balance between the aerial and the root system, as did plants produced on treatment with $5 \times 12 \mathrm{~cm}$ plant pots. Substrate composition influence was not verified. Furthermore, possible albinism and malformation in some seedlings were observed, in addition to Liriomyza sp. larvae attacks.
\end{abstract}

Key words: Pongamia pinnata, seedling quality, morphological parameters, phytosanitary problems, malformations. 


\section{INTRODUCCIÓN}

Pongamia pinnata (L.) Pierre, es una especie arbórea considerada nativa del sudeste asiático, de gran interés económico por su alto contenido de aceite en las semillas (50\% aproximadamente) que sirve para la elaboración de biodiesel, con capacidad de adaptación a tierras marginales. Puede crecer en climas húmedos a calurosos y áridos. Resiste a temperaturas desde ligeramente inferiores a $0^{\circ} \mathrm{C}$ hasta $50^{\circ} \mathrm{C}$, y precipitación anual de 250 a $2500 \mathrm{~mm}$. Se adapta tanto a suelos alcalinos, arenosos, rocosos, como salinos, crece incluso cuando la raíz está en contacto con agua salada. Estas propiedades la convierte en una fuente de energía renovable atractiva que además podría alivianar la presión que existe sobre las oleaginosas como materia prima de biodiesel (Scott et al. 2008; Ahmad et al. 2009; Biswas et al. 2013).

La calidad de plantas se define como la aptitud de los mismos para responder a los objetivos de manejo, siendo en caso de las plantaciones forestales la capacidad para adaptarse y desarrollarse a las condiciones edafoclimáticas del sitio de plantación (Mattsson 1997). Se considera que existe una relación entre los atributos morfológicos y fisiológicos de los individuos y su supervivencia y desarrollo en la plantación (Birchler et al. 1998; Tsakaldimi et al. 2012), pudiendo, de esta forma, determinar la idoneidad de los mismos mediante mediciones de algunos de estos atributos.

Por otra parte, estudios pasados han demostrado que el tamaño de maceta y la composición de sustrato son algunos de los factores que pueden influir en la calidad de plantas (Matthes-Sears et al. 1999; Olivo y Buduba 2006; Salto et al. 2013). Por lo tanto, es importante generar informaciones sobre las técnicas adecuadas para la producción de $P$. pinnata en viveros, que puedan facilitar el establecimiento a campo.
El objetivo principal es evaluar la influencia del tamaño de maceta y la composición de sustrato sobre la calidad de $P$. pinnata y adicionalmente determinar los problemas fitosanitarios y malformaciones que presentaron en el trascurso de la investigación.

\section{MATERIALES Y MÉTODOS}

El ensayo se llevó a cabo entre los meses de febrero a abril de 2010 en el Vivero Forestal de la Facultad de Ciencias Agrarias (FCA), Universidad Nacional de Asunción (UNA), ubicado en el Campus de la Ciudad de San Lorenzo, Paraguay.

El experimento se instaló utilizando un esquema factorial $3 \times 3$, consistente en la combinación de tres tamaños de macetas (M1, 5 × $12 \mathrm{~cm}$; M2, 7 × $15 \mathrm{~cm}$ y M3, 20 × 25 $\mathrm{cm})$ y tres sustratos [provenientes de suelo del orden Oxisol (S1, derivado de basalto del Departamento de Alto Paraná); suelo del orden Ultisol (S2, derivado de arenisca del Departamento Central) y suelo del orden Entisol (S3, suelo hidromórfico de la intrusión chaqueña en el Departamento Central)], considerando la posibilidad de cultivar $P$. pinnata en distintos lugares del país. En total se tuvo nueve tratamientos, los cuales fueron distribuidos completamente al azar.

Los sustratos fueron preparados mediante mezclas de los suelos mencionados anteriormente, cascarilla de coco y estiércol bovino, en proporción 2:1:1. Según el Laboratorio de Departamento de Suelos y Ordenamiento Territorial de la FCA-UNA, los tres sustratos presentaron pH levemente ácido a neutro, mediano a alto contenido de materia orgánica y macronutrientes esenciales $\left(\mathrm{P}, \mathrm{Ca}^{+2}\right.$, $\mathrm{Mg}^{+2}, \mathrm{~K}^{+}$) en mediana a alta concentración, sin embargo el contenido de $\mathrm{Na}^{+} \mathrm{y} \mathrm{Al}^{+3}+\mathrm{H}^{+}$(acidez intercambiable) fue bajo (Tabla 1). El S3 presentó el contenido más alto de $\mathrm{Ca}^{+2}, \mathrm{Mg}^{+2}$, característica propia del suelo del Chaco Húmedo y de la intrusión chaqueña.

Tabla 1. Resultados de análisis de sustratos según el Laboratorio de Departamento de Suelos y Ordenamiento Territorial de la FCA-UNA.

\begin{tabular}{|c|c|c|c|c|c|c|c|c|}
\hline \multirow[t]{2}{*}{ Sustrato } & \multirow[t]{2}{*}{$\mathbf{p H}$} & \multirow{2}{*}{$\begin{array}{c}\text { Mat. Org. } \\
\%\end{array}$} & \multirow{2}{*}{$\begin{array}{c}\text { P. } \\
\text { ppm. }\end{array}$} & \multirow{2}{*}{$\begin{array}{c}\mathbf{C a}^{+2} \\
- \\
\end{array}$} & \multirow{2}{*}{$\begin{array}{c}\mathrm{Mg}^{+2} \\
\ldots \ldots \ldots\end{array}$} & \multirow{2}{*}{$\mathbf{K}^{+}$} & \multirow{2}{*}{$\mathbf{N a}^{+}$} & \multirow{2}{*}{$\begin{array}{l}\mathrm{Al}^{+3}+\mathrm{H}^{+} \\
\end{array}$} \\
\hline & & & & & & & & \\
\hline S1 & 6,89 & 4,47 & 84,12 & 3,80 & 4,92 & 1,76 & 0,03 & 0,00 \\
\hline $\mathrm{S} 2$ & 6,50 & 2,62 & 77,65 & 3,23 & 1,99 & 0,51 & 0,00 & 0,00 \\
\hline S3 & 6,04 & 4,79 & 90,59 & 6,08 & 6,96 & 0,77 & 0,06 & 0,00 \\
\hline
\end{tabular}

Los valores de $\mathrm{pH}<5,6 ; 5,6-6,4 ; 6,5-7,4 \mathrm{y}>7,4$ corresponden a acido, ligeramente ácido, neutro y alcalino, respectivamente. Los valores de materia orgánica $<1,2 ; 1,2-2,8 \mathrm{y}>2,8$; de fósforo $(\mathrm{P})<12 ; 12-30 \mathrm{y}>30$; de calcio $\left(\mathrm{Ca}^{+2}\right)<2,51 ; 2,51-6,0 \mathrm{y}>6,0 ;$ de magnesio $\left(\mathrm{Mg} \mathrm{g}^{+2}\right)<0,4$; $0,4-0,8 \mathrm{y}>0,8$; de potasio $\left(\mathrm{K}^{+}\right)<0,12 ; 0,12-0,3 \mathrm{y}>0,3$; de Sodio $\left(\mathrm{Na}^{+}\right)<1,5 ; 1,5-3,0 \mathrm{y}>3,0$ y del ácido intercambiable $\left(\mathrm{Al}^{+3}+\mathrm{H}^{+}\right)<0,4 ; 0,4-$ $0,9 \mathrm{y}>0,9$ corresponden al nivel bajo, mediano y alto, respectivamente. 
Se utilizaron 24 individuos por tratamiento, siendo cada uno de ellos una repetición. Para su obtención, fueron sembradas 1080 semillas, una por maceta, seleccionándose para el ensayo aquellos cuyo estado de desarrollo inicial era similar. Los mismos fueron puestos bajo malla media sombra de $70 \%$ de luminosidad con una frecuencia de riego diario. La calidad de plantas se evaluó empleando los índices que combinan varias características morfológicas.

La toma de datos se llevó a cabo a los 60 días de la emergencia de las plántulas. Se determinó el índice de esbeltez para todos los individuos. Mientras que el cálculo de la relación entre el peso seco aéreo y radicular y el índice de calidad de Dickson se realizó mediante un muestreo destructivo, extrayendo en forma aleatoria ocho plantas centrales por tratamiento, para evitar el efecto de borde. Las muestras fueron secadas en estufa a $75^{\circ} \mathrm{C}$ durante 72 horas, hasta llegar al peso seco constante. Además fue registrado la presencia o no de enrollamiento de raíz en todos los individuos seleccionados.

El índice de esbeltez (IE) fue calculado mediante la relación entre la altura de la parte aérea de cada individuo (H) y su diámetro de cuello (DC) (1).

$$
\mathrm{IE}=\mathrm{H} / \mathrm{DC}
$$

$[\mathrm{cm}, \mathrm{mm}](1)$

Además se determinó la relación entre peso seco aéreo y radicular para determinar el equilibrio entre la biomasa aérea y el sistema radicular.

Se calculó también el índice de calidad de Dickson (ICD) que integra a los dos anteriores relacionando el peso seco total (PST) y la suma de la esbeltez y la relación entre el peso seco aéreo (PSA) y radicular (PSR) (Dickson et al. 1960). Este índice se empleó con éxito para predecir el comportamiento en campo de algunas especies arbóreas (Birchler et al. 1998; Sáenz et al. 2010).

$$
\mathrm{ICD}=\mathrm{PST} /(\mathrm{H} / \mathrm{DC}+\mathrm{PSA} / \mathrm{PSR}) \quad[\mathrm{g}, \mathrm{cm} / \mathrm{mm}, \mathrm{g} / \mathrm{g}](2)
$$

Para el análisis de los datos, se realizó previamente el análisis de normalidad de Shapiro-Wilk para verificar la distribución de los datos. Se aplicó el análisis de varianza y la comparación de medias a través de la prueba de medias de Tukey al nivel de $95 \%$ de probabilidad, realizando la normalización de datos para aquellos casos que la requieran. Los valores de los individuos que presentaron anomalía morfológica no fueron incluidos en los análisis estadísticos. Los gráficos fueron elaborados empleando los datos originales.

El registro de la presencia de problemas fitosanitarios y posibles malformaciones fue realizado periódicamente durante los 60 días sin considerar los tratamientos. Para estas variables no se aplicaron análisis estadísticos, limitándose a su registro y descripción.

\section{RESULTADOS Y DISCUSIÓN}

\section{Calidad de plantas}

El análisis de varianza obtenido para el índice de esbeltez fue significativo entre los niveles del factor tamaño de maceta $(p<0,05)$, no así entre los tratamientos ni entre los niveles de composición de sustrato $(p>0,05$ y $p>$ 0,05, respectivamente; Figura 1).

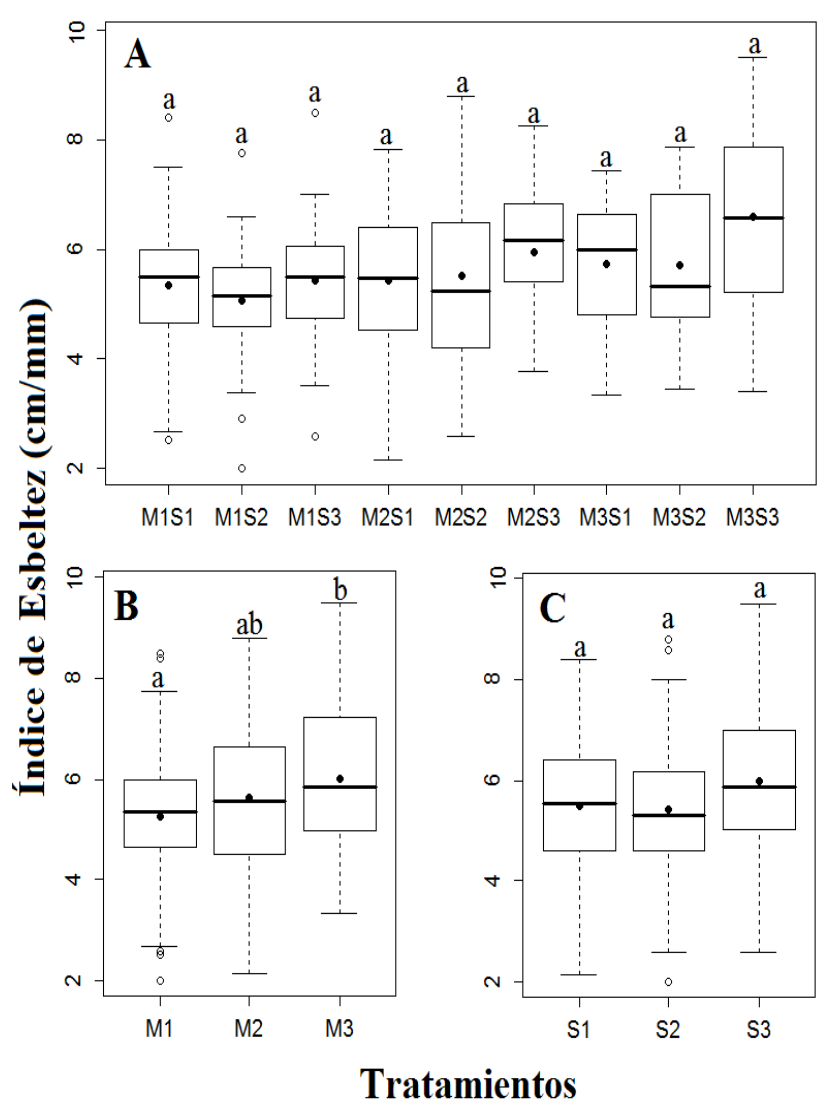

Figura 1. A. Índice de esbeltez según los tratamientos, B. Índice de esbeltez según tamaño de maceta, C. Índice de esbeltez según tipo de sustrato. Los puntos negros, los puntos blancos y las barras negras del diagrama de caja representan el promedio, valores atípicos y la mediana, respectiva mente. Las letras en cada caja representan diferencia estadísticamente significativa según la prueba de medias de Tukey a un nivel de $p=0,05$. 
De acuerdo a la prueba de comparación de medias, se formaron dos grupos entre los niveles del factor tamaño de maceta $(p<0,05$; Figura 1-B).

El índice de esbeltez más elevado y el más bajo fueron registrados en los tratamientos M3S3 y M1S2, respectivamente. Los promedios del índice de esbeltez de los individuos provenientes de los tratamientos con macetas M1, M2 y M3 fueron 5,28 $\pm 1,27 ; 5,63 \pm 1,44$ y $6,02 \pm 1,44$, respectivamente (Figura 1-B). Mientras que los promedios provenientes de los tratamientos con

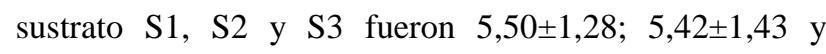
$6,01 \pm 1,46$, respectivamente (Figura 1-C).

En cuanto a la relación entre el peso seco aéreo y radicular, se obtuvo una diferencia significativa entre la media de los niveles del tamaño de maceta $(p<0,05)$; no así entre los tratamientos ni entre los niveles de composición de sustrato $(p>0,05$ y $p>0,05$, respectivamente; Figura 2).

De acuerdo a la prueba de medias de Tukey se formaron dos grupos entre los niveles de tamaño de maceta $(p<$ 0,05; Figura 2-B). Los tratamientos con macetas de tamaño 7 × $15 \mathrm{~cm}$ presentaron el menor promedio y menor desviación estándar, siendo por lo tanto los de mayor equilibrio entre el sistema aéreo y radicular.

El promedio de la relación para los tratamientos con macetas M1, M2 y M3 fueron 2,13 $\pm 0,80 ; 1,73 \pm 0,25$ y $2,25 \pm 0,52$, respectivamente. Mientras que los promedios provenientes de los tratamientos con sustrato S1, S2 y S3 fueron $1,97 \pm 0,57 ; \quad 2,13 \pm 0,74 \quad$ y $2,00 \pm 0,48$, respectivamente.

En la variable índice de Dickson se observó una diferencia significativa entre las medias de los niveles del factor tamaño de maceta $(p<0,05)$; no así entre los tratamientos y los niveles de composición de sustrato $(p>$ 0,05 у $p>0,05$, respectivamente; (Figura 3). De acuerdo con la prueba de comparación de medias, se formaron tres grupos entre los niveles de tamaño de maceta $(p<0,05$; Figura 3-B). El índice de Dickson fue directamente proporcional al tamaño del contenedor, siendo el promedio para los tratamientos con macetas M1, M2 y M3, 0,33 $\pm 0,15 ; 0,57 \pm 0,15$ y $0,90 \pm 0,34$, respectivamente (Figura 3-B). Mientras que los promedios provenientes de los tratamientos con sustrato S1, S2 y S3 fueron $0,62 \pm 0,36 ; 0,63 \pm 0,35$ y $0,56 \pm 0,29$, respectivamente (Figura 3-C).

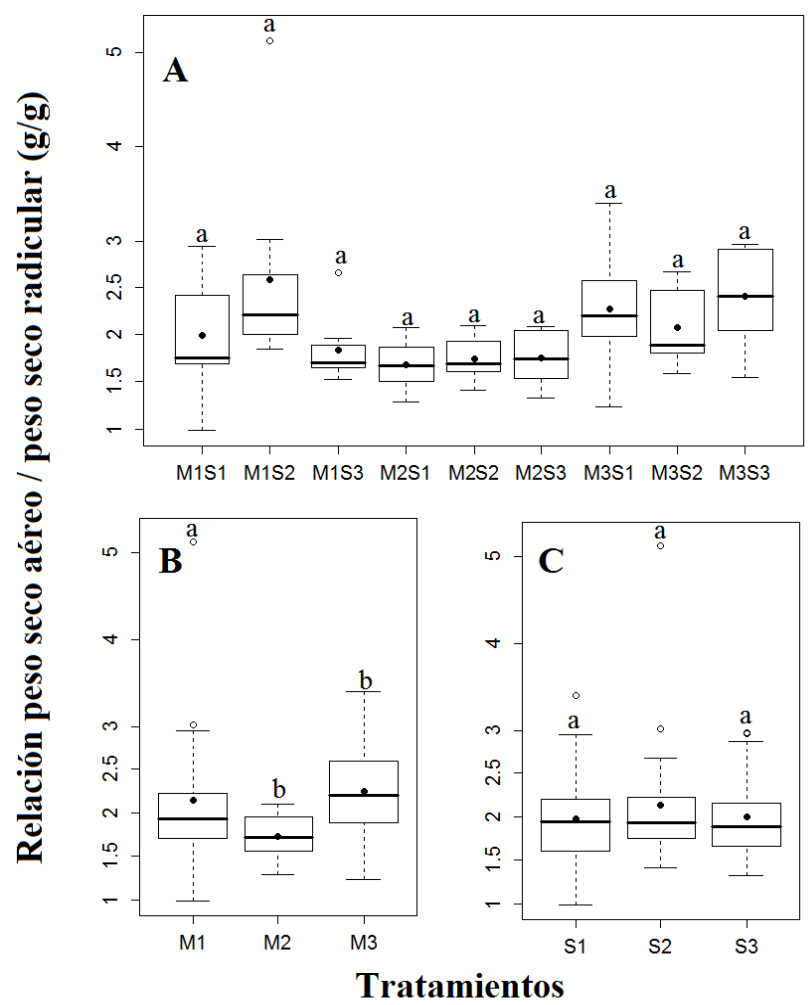

Figura 2. A. Relación entre el peso seco aéreo y radicular según tratamientos. B. Relación entre el peso seco aéreo y radicular según tamaño de maceta, C. Relación entre el peso seco aéreo y radicular según tipo de sustrato. Los puntos negros, los puntos blancos y las barras negras del diagrama de caja representan el promedio, valores atípicos y la mediana, respectivamente. Las letras en cada caja representan diferencia estadísticamente significativa según la prueba de medias de Tukey a un nivel de $p=0,05$.

Los valores medios de índice de esbeltez y relación entre peso seco aéreo y radicular (5,64 y 2,04, respectivamente) resultaron similares a los obtenidos por Mateo et al. (2011) con Cedrela odorata siendo 4,77 y 1,62, respectivamente. Mientras que el índice de Dickson reportado por estos autores fue bajo $(0,19)$ en comparación al obtenido en el presente estudio $(0,60)$, debido al elevado peso seco total de las plantas de $P$. pinnata. Olivo y Buduba (2006) reportaron valores similares a los observados en este estudio con Pinus ponderosa, siendo el índice de esbeltez, relación entre el peso seco aéreo y radicular e índice de Dickson 4,98 (equivalente a 1,05 en índice de esbeltez de SchmidtVogt); 1,59 y 0,72 , respectivamente. 


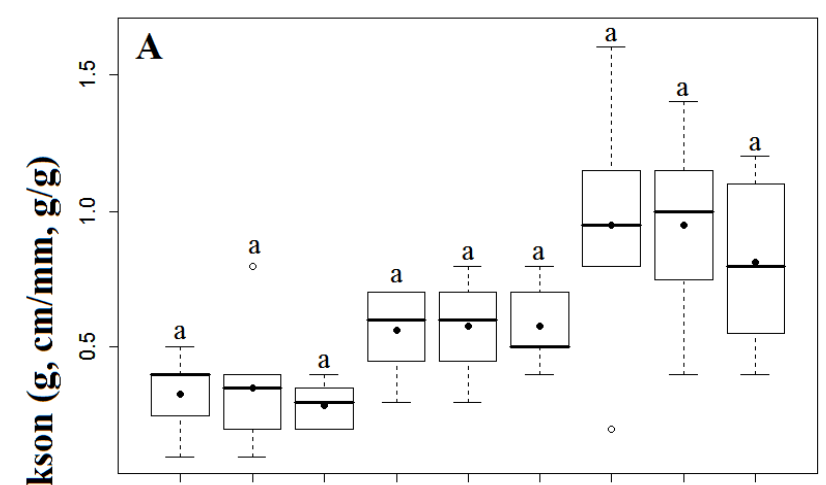

M1S1 M1S2 M1S3 M2S1 M2S2 M2S3 M3S1 M3S2 M3S3
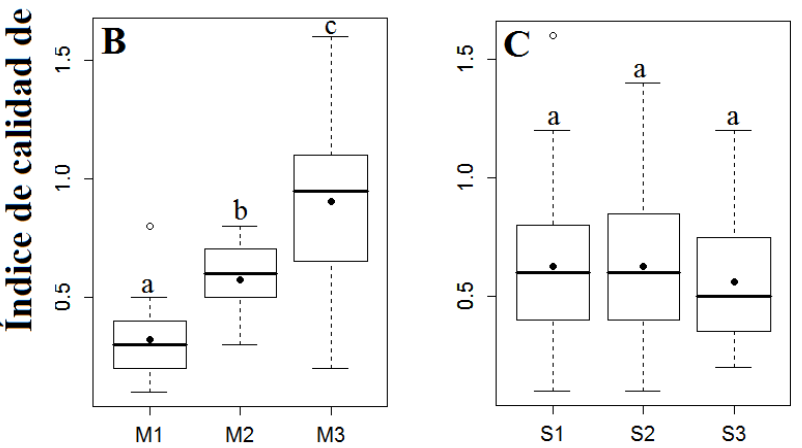

Tratamientos

Figura 3. A. Índice de calidad de Dickson según los tratamientos. B. Índice de calidad de Dickson según tamaño de maceta, $\mathbf{C}$. Índice de calidad de Dickson según tipo de sustrato. Los puntos negros, los puntos blancos y las barras negras del diagrama de caja representan el promedio, valores atípicos y la mediana, respectiva mente. Las letras en cada caja representan diferencia estadísticamente significativa según la prueba de medias de Tukey a un nivel de $p$ $=0,05$.

Dickson et al. (1960) menciona que los valores más altos del índice de Dickson indican plantas de mejor calidad. Sin embargo, a juzgar mediante los resultados obtenidos, en el presente estudio no se cumplió esta relación. El peso seco total fue el factor de mayor ponderación en el valor de este índice, impidiendo que se refleje la incidencia del equilibrio entre el peso seco aéreo y radicular.

Se considera que los tratamientos con macetas de tamaño 7 x $15 \mathrm{~cm}$ (M2S1, M2S2 y M2S3) fueron los de mejor calidad. A pesar de presentar un valor intermedio de índice de calidad de Dickson $(0,57)$, estos presentaron mejor equilibro entre el peso seco aéreo y radicular $(1,73)$ (Figura 2 y 3 ).
Los tratamientos con macetas de tamaño $20 \times 25 \mathrm{~cm}$ (M3S1, M3S2 y M3S3) no pueden ser considerados de mejor calidad, a pesar de los elevados valores del índice de calidad Dickson $(0,90)$, debido al menor equilibrio entre peso seco aéreo y radicular (2,25) (Figura 2 y 3). El reducido peso seco radicular en comparación al aéreo puede deberse a que la altura de la maceta $(25 \mathrm{~cm})$ en relación a su diámetro $(20 \mathrm{~cm})$ no fue la adecuada para el desarrollo vertical del sistema radicular. La presencia de enrollamiento de raíz en todos los individuos muestreados del M3S1, M3S2 y M3S3 $(N=24)$ confirman este argumento (Figura 4-a). Estos resultados indican la importancia de la dimensión (relación entre la altura y la anchura) de la maceta para el desarrollo de los individuos.

Es probable que la composición de sustrato no haya incidido significativamente en la calidad de las plantas debido tanto a la disponibilidad de nutrientes en las tres composiciones como a su alta plasticidad.

Generalmente se asume que las plantas de mejor calidad presentan mayor valor del índice de Dickson (Dickson et al. 1960). Sin embargo, considerando que las características morfológicas podrían variar entre las especies, se recomienda determinar el rango apropiado del índice para $P$. pinnata. Los resultados del presente estudio indican que el rango idóneo del índice de calidad de Dickson para $P$. pinnata podría estar en torno a 0,50 , debido a que los tratamientos con mejor equilibrio entre peso seco aéreo y radicular presentaron un promedio de 0,57 . Es necesario evaluar el desempeño de estos individuos en el campo para comprobar lo expuesto.

\section{Ataque de insectos y malformaciones}

Se registraron ataques de insectos del Orden Diptera, Familia Agromyzidae, genero Liriomyza, conocida como mosca minadora, que son pequeñas moscas de color verde metálico a azulado que ovipositaban en las hojas más tiernas de P. pinnata (Figura 4-b). Una vez eclosionadas, las larvas empezaban a minar las hojas, consumiendo el mesófilo y dejaban intacta las capas epidérmicas. Las minas así producidas eran de forma irregular. Las larvas eran de color amarillo, de unos $3 \mathrm{~mm}$, ápodas y cilíndricas, con gancho mandibular claramente observable en el estereoscopio. El 11\% de las plantas estudiadas $(N=$ 216) sufrieron ataques de larvas de esta mosca.

Se registraron deformaciones en el ápice terminal en cuatro de los 216 individuos empleados en el ensayo (Figura 4-d). Este problema no causó la muerte de las 
plantas, pero inhibió o retrasó el crecimiento.

También fue identificada la presencia de individuos albinos. De las 1080 semillas sembradas, se obtuvieron dos individuos albinos (Figura 4-c). Cabe destacar que estos individuos no fueron incluidos en el ensayo de calidad de plantas. La presencia de albinos se debe a la carencia de clorofila. Estos individuos no sobrevivieron por mucho tiempo, se secaron cuando se agotaron la reserva de nutrientes de los cotiledones. Estudio realizado en el estado de Orissa, India reportó la aparición de 2,5\% de plantas albinos (Mohanty et al. 2005). Cabe destacar que las semillas empleadas en el presente estudio provienen de Chhattisgarh, estado lindante con Orissa.

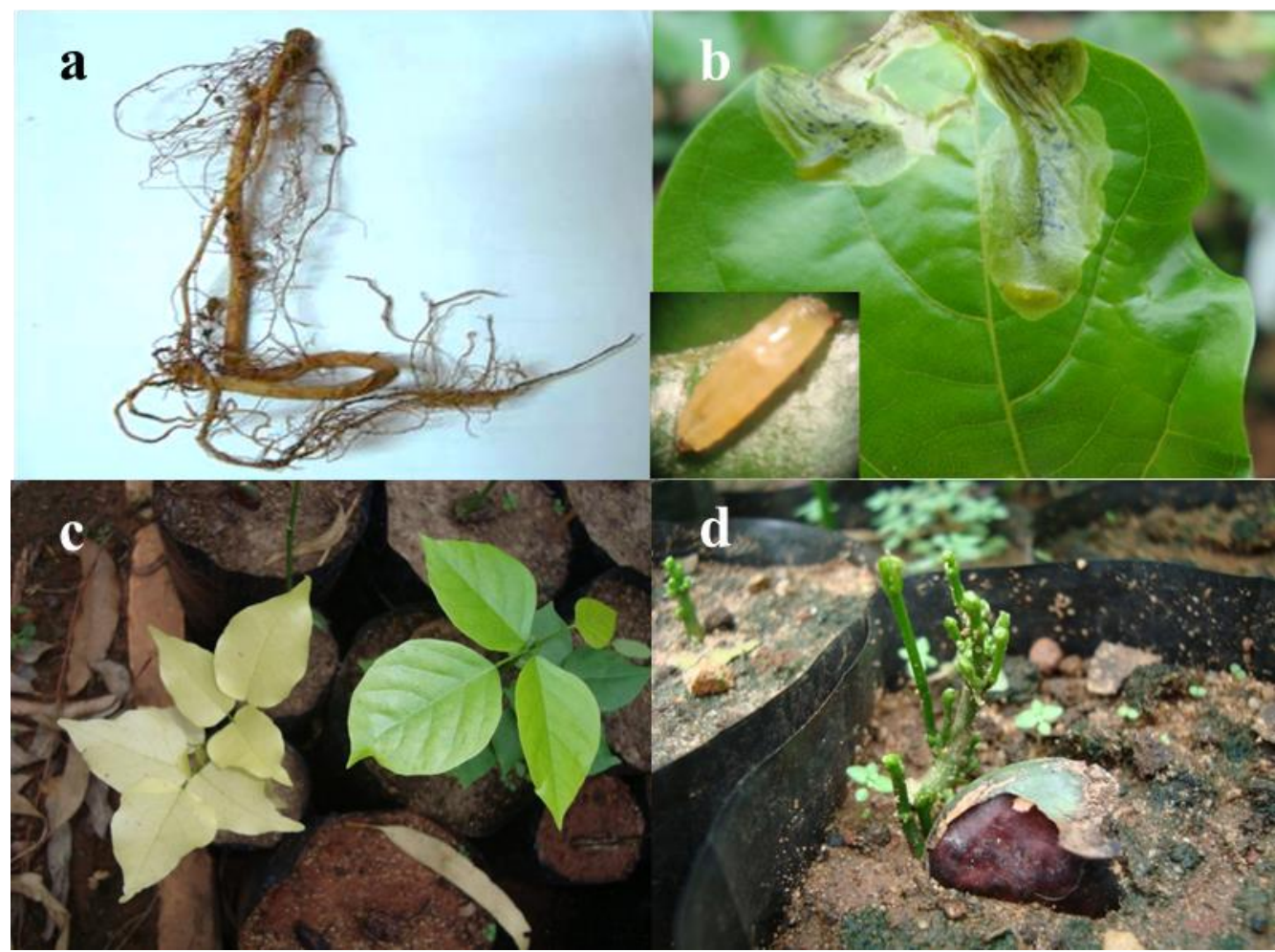

Figura 4. a. Enrollamiento de raíz y formación de nódulos en los individuos, b. ataque de larvas de Liriomyza sp., c. individuo albino (izquierda) y con clorofila (derecha), d. individuo con deformaciones o achicharramiento del ápice.

\section{CONCLUSIONES}

Independientemente de la composición del sustrato, los tratamientos con macetas de tamaño 7 x $15 \mathrm{~cm}$ presentan plantas de mejor calidad considerando que tienen mayor equilibrio entre peso seco aéreo y radicular. No se verifica la influencia de la composición de sustrato sobre las variables evaluadas. Se registran ataques de Liriomyza sp., presencia de posibles malformaciones y albinismo.

\section{REFERENCIAS BIBLIOGRÁFICAS}

Ahmad, M; Zafar, M; Khan, MA; Sultana, S. 2009. Biodiesel from Pongamia pinnata L. oil: a promising alternative bioenergy source. Energy Sources, part A: recovery, utilization, and environmental effects 31(16): 1436-1442.

Birchler, T; Rose, RW; Royo, A; Pardos, M. 1998. La planta ideal: revisión del concepto, parámetros definitorios e implementación práctica. Investigación Agraria 7(1-2): 109-121.

Biswas, B; Kazakoff, SH; Jiang, Q; Samuel, S; Gresshoff, PM; Scott, PT. 2013. Genetic and genomic analysis of the tree legume Pongamia pinnata as a feedstock for biofuels. The Plant Genome 6(3): 1-5. 
Dickson, A; Leaf, AL; Hosner, JF. 1960. Quality appraisal of white spruce and white pine seedling stock in nurseries. Forest Chronicle 36: 10-13.

Matthes-Sears, U; Larson DW. 1999. Growth and survival by the quantity and quality of rooting space: implications for the establishment of Thuja occidentalis on cliff faces. International Journal of Plant Sciences 160 (1): 122-128.

Mattsson, A. 1997. Predicting field performance using seedling quality assessment. New Forests 13 (1-3): 227-252.

Mateo Sánchez, JJ; Bonifacio Vázquez, R; Pérez-Ríos, SR; Mohedano Caballero, L; Capulín Grande, J. 2011. Producción de (Cedrela odorata L.), en sustrato a base de aserrín crudo en sistema tecnificado en Tecpan de Galeana, Guerrero, México. Ra Ximhai 7(1): 123-132.

Mohanty, M; Bhola, N; Mohanty, S. 2005. Albino seedlings in Pongamia pinnata (L.) Pierre. Indian Forester 131 (1): 127-128.
Olivo, VB; Buduba, CG. 2006. Influencia de seis substratos en el crecimiento de Pinus ponderosa producido en contenedores bajo condiciones de invernáculo. Bosque 27(3): 267-271.

Sáenz, RJT; Villaseñor, RFJ; Muñoz, FHJ; Rueda, SA; Prieto, RJA. 2010. Calidad de planta en viveros forestales de clima templado en Michoacán. Folleto Técnico Núm. 17. Uruapan, Michoacán, MX, SAGARPA. 48 p.

Salto, CS; García, MA; Harrand, L. 2013. Influencia de diferentes sustratos y contenedores sobre variables morfológicas de plantines de dos especies de Prosopis. Quebracho 21(1-2): 90-102.

Scott, PT; Pregelj, L; Chem, N, Hadler, JS: Djordjevic, MA; Gresshoff, PM. 2008. Pongamia pinnata: an untapped resource for the biofuels industry of the future. Bioenergy 1: 2-11.

Tsakaldimi, M; Ganatsas, P; Jacobs, DF. 2012. Prediction of planted seedling survival of five mediterranean species based on initial seedling morphology. New forests 44(3): 327-339. 